Please quote as: Mrass, V.; Peters, C. \& Leimeister, J. M. (2018): Managing Complex Work Systems via Crowdworking Platforms: How Intel and Hyve Explore Future Technological Innovations. In: Hawaii International Conference on System Sciences (HICSS), Doctoral Consortium. Waikoloa, HI, USA. 


\section{Managing Complex Work Systems via Crowdworking Platforms: How Intel and Hyve Explore Future Technological Innovations}

\author{
Volkmar Mrass \\ University of Kassel \\ volkmar.mrass@uni-kassel.de
}

\author{
Christoph Peters \\ University of St.Gallen \\ and University of Kassel \\ christoph.peters@unisg.ch
}

\author{
Jan Marco Leimeister \\ University of St.Gallen \\ and University of Kassel \\ janmarco.leimeister@unisg.ch
}

\begin{abstract}
Crowdsourcing has the potential to change the way how companies and other organizations are working currently. Numerous companies are already exploiting this new form of work organization and are utilising the "wisdom of crowds". Crowdworking platforms as intermediaries that manage the work system including customer companies and crowd workers play an important role in this context. Nevertheless, they currently mostly manage rather simple work systems that process rather plain work. In this summary for the HICSS 2018 Doctoral Consortium, we depict our current work in progress that aims at investigating how such platforms could also manage more complex work systems - a question that is crucial for the future success of this business model. Using the case of Intel and the crowdworking platform Hyve, we investigate one successful approach to tackle this challenge, elaborate on our method used as well as the theoretical background and communicate our first, preliminary findings.
\end{abstract}

\section{Introduction}

According to the HICSS 2018 DC instructions, this 5-pages summary of the research should begin with the research question and state why it is important. Our research question is:

RQ: How can complex work systems successfully be managed via crowdworking platforms?

From our perspective, there are at least three reasons why it is important to investigate this issue: First, the increasing use of artificial intelligence (AI). This will lead to the replacement of plenty of work currently conducted e.g. on microtask platforms that at the moment still requires human intelligence. To be able to process also more complex work will be paramount for the future of the business model of crowdworking platforms. Second, the fact that societal players and legislators in several countries worldwide (see e.g. [2]) are discussing issues such as minimum wage requirements or working conditions in general on such platforms. The more complex work is, the more likely it is that this work will result in higher payments that meet possible future legal requirements. Third, the business model of crowdworking platforms simply offers more potential than currently used. Utilising this potential could lead to more orders and increased revenues for the crowdworking platforms and to both advantages in realms such as costs, efficiency and speed for the ordering companies respectively to new employment possibilities for individuals. Besides these reasons for certain stakeholder groups such as crowdworking platform providers, companies and individuals, this question is also important for economies and societies as a whole: The continuing digitization also greatly affects countries, their economies and their competive advantage [8]; using innovative new 'instruments' such as crowdworking platforms can result in significant benefits in this competition.

To gain insights to answer this research question, we investigate several seminal projects from different customers processed via different crowdworking platforms with the aim to extract measures that foster the successful management of complex work systems via crowdworking platforms. All of the projects we selected and that cover different industries proved to be very successful and therefore likely to provide insights for our research question. One project dealt with the development of services for a worldwide leading bank, another project with the exploration of new opportunities for one of Europe's largest local public transportation companies. With the research in progress we depict in this paper, we focus on a project from the semiconductor industry: the case of Intel and the innovation crowdworking platform Hyve. This project had the goal to find possible future applications for a new and innovative sensor technology that is planned to be released in the course of the year 2017.

\section{Theoretical Background}

Work systems managed via crowdworking platforms differ regarding several aspects from those in 'regular' organizations: One is the fact that work is usually processed on and supported by electronic platforms and mostly does not take place "face-to-face" in the "analogue" world. Furthermore, companies often do not know the workers who process their work, nor do these workers usually know each other. In addition, crowd 
workers on such platforms have very heterogenous educational and professional backgrounds and skills and the platforms themselves foster the processment of quite heterogenous kinds of work ([9]). The crowd workers are also often distributed among different countries and time zones. Another main characteristic of work systems managed via crowdworking platforms that differs from that in 'regular' companies is the paradigmatic change that work is not assigned to workers anymore, they rather choose their work themselves ([10]).

In general, crowdworking platforms can be seen as intermediaries and the point where the controlling and management of the crowd and of all activities within the crowd take place [7]. If these platforms focus on the performance of paid work, it is reasonable to use the term crowdworking platforms ([11]) as a subset of crowdsourcing platforms (the latter also include for example platforms for crowdfunding or crowdvoting). In organizational environments, work is "the application of human, informational, physical, and other resources to produce products/services" ([1], p. 75). Complex work is for the authors of this paper - derived from the description of characteristics of simple work on a microtask platform [5] and reversing these - in general work that mostly requires coordination, a high level of cognitive effort, expertise and skills in the respective area, time and contextual information; it is usually heterogeneous, interdependent, rather non-repetitive and has multiple stakeholders. The World Bank uses in a study [6] skills and education or training required as a proxy to determine work complexity. It assigns low complexity to microwork where mostly no specialized skills or training are required and basic computer and
Internet literacy (and the associated language) skills are usually sufficient. It attributes high complexity ([6], p. 13) to work from areas such as engineering, software development or human resources.

A work system is "a system in which human participants and/or machines perform work (processes and activities) using information, technology, and other resources to produce specific products/services for specific internal and/or external customers" ([1], p. 75). In our case, participants of the work system are first of all the crowd workers (assembled by the crowdworking platform Hyve) who provided the solution ideas. But also customer representatives (of Intel) since they participated in the creation of the idea solutions for products and services and internal employees of the platform operator Hyve, especially those who have been occupied with the crowd community management.

Information refers to informational entities such as orders or invoices as well as to conversations and verbal commitments by the work system participants ([1], p.80). Technologies include both tools that are used by work system participants and automated agents (i.e. hardware and software configurations) since some work systems are totally automated (ibidem). Processes and activities occur in the work system to create products and services for its customers. Besides the elements of the work system itself described above, the Work System Framework ([1], p. 78) also includes environment, infrastructure and strategies. We believe that the complexity of work is a good proxy for the complexity of the work system necessary to process that work. With our research, we are looking at IT-reliant work systems; more specifically, at work systems that are managed via

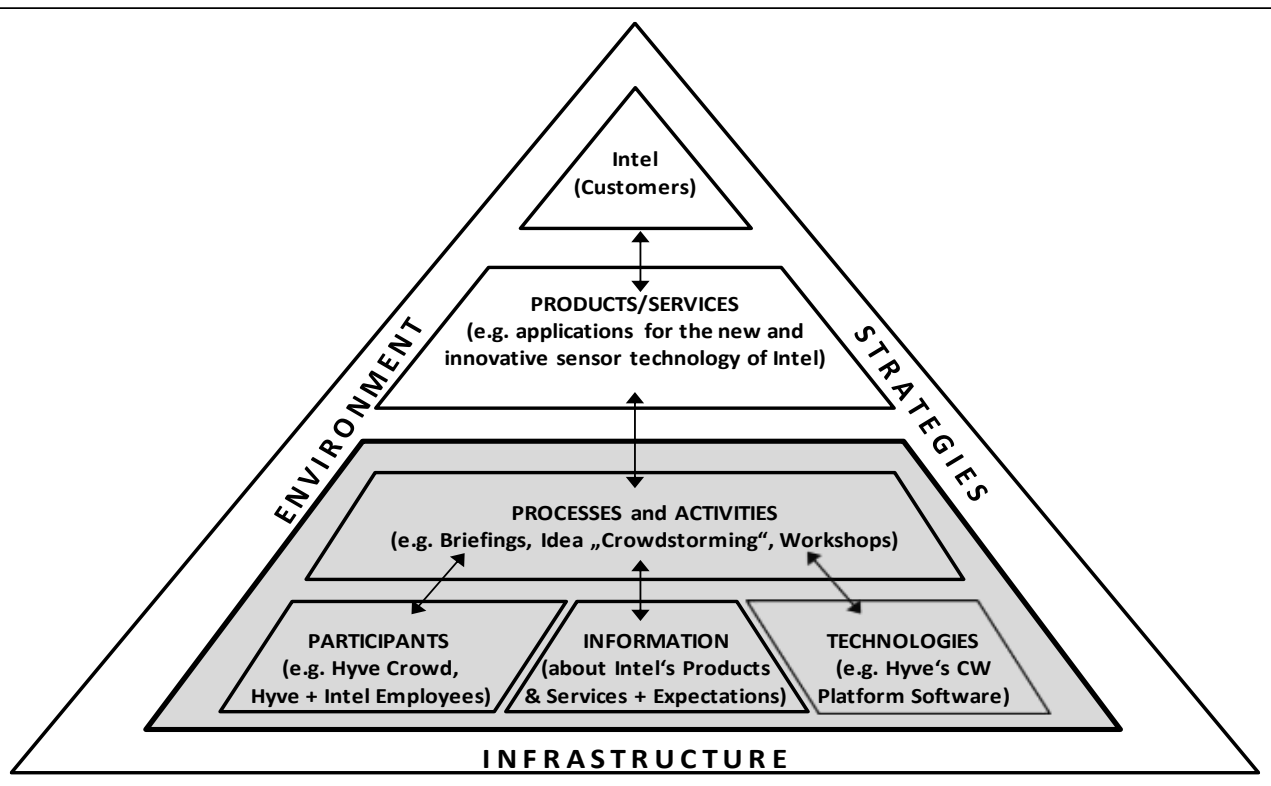

Figure 1. Depiction of the Intel \& Hyve Work System based on Alter ([1], p. 78) 
crowdworking platforms. We purposefully mostly use the term "manage" (in the sense of planning/steering/controlling) instead of "govern" since the landscape of crowdworking platforms is heterogeneous and some platforms are more coordinating the supply and demand than governing the whole work system (although the term govern would be accurate in many cases). Figure 1 depicts the result of our analysis of the work system in place regarding this project of Intel and Hyve.

\section{Methodology and Case Selection}

Since we pursue a "how?"-question, we employ the research method 'case study' that according to Yin [12] is especially adequate and useful for such issues. $\mathrm{He}$ states that the case study research method is in general especially useful when (1) the main research questions are "how" or "why" questions, (2) a researcher has little or no control over behavioral events and (3) the focus of study is a contemporary (not entirely historical) phenomenon. This is true regarding our investigation: With our research question, we strive to examine how complex work systems can be managed via crowdworking platforms; we have no influence on behavioral events since we do not interfere in the interactions between crowdsourcers, platforms and crowd workers and the focus of our study, crowdworking platforms, are a current phenomenon and not something from the past. Investigating the unit of analysis of our research - the work system consisting of processes and activities, participants, information and technologies - aims at providing valuable insights and sheding light on this issue. In order to realize triangulation and thereby providing a stronger substantiation of constructs and hypotheses as suggested by Eisenhardt [4], we used the following multiple sources to collect data for this case study so far:

- In-depth interview with Markus Rieger, Managing Director of Hyve on site at Hyve's headquarters in Munich (on May $31^{\text {st }}, 2017$ )

- In-depth interview with Dr. Johannes Gebauer, Senior Innovation Consultant of Hyve and project leader of the "Intel Future Contest" project (telephone conference) (on July $5^{\text {th }}, 2017$ )

- Several contacts with and information from further employees of Hyve (March 2017)

- Analysis of the publicly available information about the Intel \& Hyve project (May to July 2017)

- Analysis of the information available on Intel's and Hyve's Internet websites (May 2017)

- Evaluation of additional material received from Hyve after the interviews.

For the semi-structured interviews, we developed a guideline with questions addressing different areas of the work system (e.g. measures for the steering of the participants, the planing of the processes/activities, the design of the platform/technology etc.) to get insights from different perspectives on the theme of managing complex work systems via crowdworking platforms such as Hyve. The interviews were recorded and subsequently transcribed. A draft of the resulting intermediate paper has been delivered to the interviewees to offer the possibility to comment it or request adjustments if contentwise necessary.

To be able to generate the desired insights, we chose a crowdworking platform respectively a project that:

- Deals with the management of complex work (systems) as defined in section 2

- Is positioned on an international basis, i.e., has crowd workers and customers from all over the world (to ease comparability and the application of the findings on an international level)

- Has already been existing for a while (i.e., at least three years) and shows a good business record (i.e., high revenues), therefore making it more likely that it has gained enough expertise to answer our questions regarding the management of complex work systems adequately

- Has conducted projects that have been very successful (to be able to generate useful recommendations for projects of other companies and platforms).

We selected the crowdworking platform "Hyve" and its project with Intel since it provides plenty of insights on the management of complex work systems. Hyve also allows the gearing of both external and internal (employees of a company) crowds - a measure that has been identified as a key for the successful management of complex work systems via crowdworking platforms in earlier research [11]. The platform is furthermore not restricted to the use in a specific company (which is the case with several proprietary platforms), but open to companies worldwide that want to use its services. Despite the fact that the area of crowdworking companies is dynamic and many companies that existed years ago do not exist anymore (e.g. because they merged with other companies or went bankrupt), Hyve has already been on the market for more than a decade, generates high revenues, and proved to be able to "survive" in the long run. Moreover, the areas of technological services and innovation are in general areas that require the management of more complex work systems than it is for example the case regarding microtask platforms. Therefore, the examination of the seminal project managed via the crowdworking platform Hyve with Intel proved to be very valuable to answer our research question. 


\section{The Case of Intel and Hyve}

\subsection{Company Profiles and Project}

Intel Corporation is one of the world's largest semiconductor chip manufactors. Founded in 1968 and based with its headquarters in Santa Clara/California (United States), the company employs more than 100,000 people and generates revenues of over 50 billion USD (see also: www.intel.com/content/www/us/en/companyoverview/company-overview.html). Since 1974, Intel has its European headquarters in Munich. In the realm of PC microprocessors, Intel is the leading company in the world with a worldwide market share of over $80 \%$.

Hyve is an innovation company that is headquartered in Munich/Bavaria (Germany), employs about 100 people and gathered a crowd community of about 30,000. Founded in 2000 and conducting crowd contests since 2008 , the company offers a crowdworking platform and co-creates products and services with consumers, lead users and experts from more than 140 countries. Over $70 \%$ of the DAX (main German Stock Index) companies are among Hyve's customers (see also: https://www.hyve.net/en/about/ and www.linkedin.com/company/hyve-ag).

In 2012, Intel approached Hyve for the conduction of a "Future Contest" that aimed at exploring its new sensing technology and finding innovative applications and use cases for it. The impulse came from experts from Intel Israel who approached their colleagues at the Intel headquarters in the United States who then subsequently approached Hyve in Germany. The new sensing technology remembers a person's surrounding by hearing and seeing and transforms the gained information into data such as pictures or videos. Hyve and Intel subsequently launched a contest via Hyve's crowdworking platform with the goal to gain ideas and solutions for use cases. On the whole, 1,613 members on the platform submitted a total of 488 ideas including detailed visualizations. This resulted in plenty of new suggestions how to implement the new sensing technologie into daily life. The launch for this technology is planned to take place in 2017 (see also: www.hyve.net/en/work/references/intel-future-contest/).

\subsection{Project Execution and Insights}

The close and thorough analysis of this seminal project of Intel and Hyve and the general proceeding of Hyve regarding the management of complex work systems provided us with valuable insights for our research question. Hyve usually takes the following measures to ensure the successful completion of a complex project: At the beginning, Hyve first conducts a conception workshop of about four to eight hours with the customer
- depending on the complexity of the issue. About 50 percent of this workshop is devoted to define and formulate the theme - this is paramount to get the crowd involved and allow them to closely understand the issue. Afterwards, Hyve develops a recruiting campaign with the customer and defines its target groups (e.g. students, engineers, designers, etc.) as well as the channels to reach out to them. For the project with Intel, Hyve for example also used social media channels such as Facebook and Twitter. Together with the customer, Hyve also determines what prizes are advertized, how the process should look like and if their crowdworking platform should use any branding elemtens (e.g. the logo of the customer company) for the respective contest/project. The latter is done often since many customer companies also use such contests as marketing instruments to promote their brand. Afterwards, the project goes "live" on the platform. Hyve never starts an "empty" contest, but instead generates some initial ideas to foster discussions. The "live" phase on the crowdworking platform usually lasts between 6 and 10 weeks (in the case of Intel from November $6^{\text {th }}, 2012$, to December $18^{\text {th }}$, 2012). Hyve employs an own "campaigning" team of 12 internal employees who are occupied with moderating and managing such a project. This community management by Hyve also includes to welcome every participant individually (with a personalized message using his/her name). The number of participants from the crowd differs with around 10,000 crowd workers participating in projects with very well-known brands such as for example Daimler/Mercedes-Benz.

Hyve reads the incoming ideas and solutions, comments them, provides feedback or routes inquiries to the responsible employee of the customer if an information is necessary to proceed. Having for example experts from Intel comment their ideas and solutions proved to be a good motivation for Hyve's crowd. Hyve emphasizes the aspect of collaboration within this work system that is paramount to further develop initial ideas and solutions and for a successul management of complex work systems on the whole. Also paramount: The close support for the customer that Hyve for example ensures by updating him with weekly reports about the proceeding of the contest and project including benchmarks to past contents and projects. After the 6 to 10 weeks of “crowdstorming" via Hyve's crowdworking platform, there are usually several hundred ideas that now have to be shortlisted. Then, Hyve conducts an expert workshop with the customer where three or four experts are invited who prepare the jury meeting. After this workshop, about 10 to 15 ideas go into the jury meeting. In the jury meeting, the winners of the contest are selected and the awards assigned (usually prizes of about 3,000, 2,000 and 1,000 Euro each for the first three winners). The selection of the winners is based on several criteria such 
as best evaluation from the crowd community, best idea based on the jury's judgement, most comments during the crowdstorming, and more. These winners are later not necessarily also the participants which whom the customer later wants to further develop an idea. In general, Hyve and the customer do not further develop single ideas, but cluster of such ideas. Hyve also often accompanies the customer further to develop prototypes. Sometimes, Hyve also conducts subsequent ideation workshops with the winners and several experts and develops concepts that then might be patented and afterwards implemented. Hyve sometimes (like in the case of a project with Deutsche Telekom) accompanies the whole process including the serial production.

This approach depicted above proofed also to be successful for the management of the work system in the project with Intel. Even though this project with Intel is regarded as one of the most complex Hyve had conducted so far, the crowd was able to deliver plenty of new interesting ideas and stimuli for possible use cases of Intel's new sensing technology. The jury including representatives of Intel has been both impressed and surprised by the performance and depths of solutions. The project resulted in ideas that Intel did not have "on their radar" and that have the potential to make a big impact. The winning ideas covered different areas - from entertainment over healthcare to security.

\section{$5 \quad$ Preliminary Findings}

From this seminal case, we are able to derive several important measures for the successful management of complex work systems via crowdworking platforms as a new form of work organization. Surprinsingly, the crowdworking platform software itself is according both CEO Markus Rieger and senior consultant Dr. Johannes Gebauer from Hyve not a key factor. It is rather a mere "tool" that must function, where the ideas and solutions are entered and stored, but that only can affect results negatively if it does not work properly:

"Software alone is not the major success driver, quite the contrary: If I as a company would have the choice to take the best software of the world without professional campaign management support or to take a Google-Doc-list with professional support, I would absolutely take the latter. Because even with that for a crowd contest rather impractical tool, the results would be better since the management of the whole process is the key to success."

Markus Rieger, Managing Director of Hyve

The management of the process via Hyve (depicted in detail in section 4.2), including close coordination with the customer, community management for the crowd and gearing of external contributors and internal employees of the customer proofed to be one of the key success factors. Actively integrating experts from Intel who provided comments and suggestions on interesting ideas and solutions from the crowd helped to push the quality of results from the beginning. These experts for example communicated in an early stage when an idea has been interesting, but did not fit the strategy of the customer or would not be possible to implement due to technical restrictions. The community management via Hyve's internal employees has been important, too. The heterogeneity of the crowd workers in this project from more than 60 nations and different educational backgrounds could have led to different interpretations of the theme. But the criteria, comments and feedback provided during the process helped to make the desired solution more clear. Transparency about the goals and criteria is crucial for a project to get in the right direction.

Even though the process is managed via an electronic (crowdworking) platform, there seems to be a trend to also conduct several steps "offline" when it comes to the management of complex work systems. Hyve for example goes to universities and invites students to conduct casual evening gatherings (with "beer and pizza") where they generate for two hours ideas and simultaneously enter them into the Hyve tool. The same is true when it comes to issues that are very sensitive regarding confidentiality. Hyve then conducts on site "leaduser workshops" with about ten people who have to sign non-disclosure agreements. The crowds that process work can therefore be "hand-picked" and small. This leads to another derived measure from this case: Targeting selected groups from the crowd for certain projects. Hyve for example does - with the exception of some hundred power users who have allready participated in plenty of projects - not contact all crowd workers for every project, but those who match the respective requirements well.

Besides financial incentives, non-financial incentives also play an important role when it comes to the management of complex work systems: Hyve emphasized that for example the fact that there is a top-tier jury of renowned personalities (ideally including members of the management board of the customer company) does also have an effect on the crowd and results in both a higher number of participants and more highly qualified participants that in turn positively influences the quality of the results. At the same time, there is the risk of "too many ideas" (see also in the literature [3]):

"The crucial value does not necessarily come directly from a few of the 500 or 5,000 single ideas. The sheer mass of ideas is of limited use for the customer. The trick is to read between the lines, connect the ideas and derive superior concepts based on the multilayered input we receive from the community." Dr. Johannes Gebauer, Senior Innovation Consultant of Hyve 
Another measure for the successful management of complex work systems via crowdworking platforms that can be derived from the case of Intel and Hyve is that crowdworking platforms should not only deliver the results from the crowd contest on their platform to the customer, but also to further accompany him in the further process - ideally untill the execution and implementation. In this context, we also found that the approach to divide work, distribute it among several workers or process it and afterwards reassemble it does not work for complex work systems such as that of Intel and Hyve.

\section{Acknowledgements}

This paper presents research that was conducted in the context of the project "Challenge cloud and crowd" that is funded by the German Federal Ministry of Education and Research (BMBF) within the program "Innovations for Tomorrow's Production, Services, and Work" (funding number: 02K14A071) and managed by the Project Management Agency Karlsruhe (PTKA).

\section{References}

[1] Alter, S. 2013. Work System Theory: Overview of Core Concepts, Extensions, and Challenges for the Future. Journal of the Association for Information Systems (JAIS) 14, 2, 72-121.

[2] Benner, C., Ed. 2014. Crowdwork - zurück in die Zukunft? Perspektiven digitaler Arbeit. Bund-Verlag $\mathrm{GmbH}$, Frankfurt am Main.

[3] Blohm, I., Leimeister, J. M., and Krcmar, H. 2013. Crowdsourcing: How to Benefit from (Too) Many Great Ideas. MIS Quarterly Executive 12, 4, 199-211.

[4] Eisenhardt, K. M. 1989. Building Theories from Case Study Research. The Academy of Management Review $14,4,532-550$.

[5] Kittur, A., Smus, B., Khamkar, S., and Kraut, R. E. 2011. CrowdForge: Crowdsourcing Complex Work. Proceedings of the 24th annual ACM Symposium on User Interface Software and Technology: October 1619, 2011, Santa Barbara, CA/USA.

[6] Kuek, S. C., Paradi-Guilford, C. M., Fayomi, T., Imaizumi, S., and Ipeirotis, P. 2015. The Global Opportunity in Online Outsourcing. The World Bank, Washington.

[7] Leimeister, J. M. and Zogaj, S. 2013. Neue Arbeitsorganisation durch Crowdsourcing. Eine Literaturstudie. Arbeitspapier 287, Düsseldorf.

[8] Mrass, V., Li, M. M., and Peters, C. 2017. Towards a Taxonomy of Digital Work. 25. European Conference on Information Systems (ECIS), Guimarães, Portugal, 2017.

[9] Mrass, V. and Peters, C. 2017. Crowdworking-Plattformen in Deutschland. Working Paper Series 16. University of Kassel, Kassel.
[10] Mrass, V., Peters, C., and Leimeister, J. M. 2016. New Work Organization through Crowdworking Platforms. A Case Study. Konferenz, "Zukunftsprojekt Arbeitswelt 4.0", 19.09.2016, Stuttgart.

[11] Mrass, V., Peters, C., and Leimeister, J. M. 2017. One for All? Managing External and Internal Crowds through a Single Platform - A Case Study. 50th Hawaii International Conference on System Sciences (HICSS), 2017, 4324-4333.

[12] Yin, R. K. 2014. Case Study Research. Design and Methods. SAGE Publications, Inc., Los Angeles. 